\title{
A TRACE PRESERVING OPERATOR AND APPLICATIONS
}

\author{
MENITA CAROZZA, JAN KRISTENSEN, AND ANTONIA PASSARELLI DI NAPOLI
}

\begin{abstract}
We construct a trace preserving operator which improves the integrability of functions in Sobolev classes refining the ones available in literature. As applications, we prove a $C^{1, \alpha}$ partial regularity result for local minimizers of quasiconvex integral functionals satisfying non standard $(p, q)$ growth conditions in the borderline case $p=n-1$ and $q=n$, and a global integrability result for weak solutions to a nonlinear elliptic system.
\end{abstract}

AMS Classifications. 49N15; 49N60; 49N99.

Key words. Trace operator; non standard growth; partial regularity.

\section{InTROdUCTION AND STATEMENTS}

The aim of this paper is to construct a linear operator from $W^{1, p}$ into $W^{1, p}$ which preserves the boundary values and improves the integrability of the functions and of their distributional gradients. A suitable regularity assumption on the boundary allows us to obtain an improved integrability result that, in certain aspects, refines those available in literature (see in particular [23]). Our construction is similar to an approach used by Gagliardo and we obtain the integral bounds expoliting an idea used by Hedberg for proving boundedness of Riesz potentials. It relies on a convolution kernel operator that leads us to extend a function defined on $\mathbb{R}^{n-1}$, considered as the boundary hyperplane of the $n$-dimensional upper half space $\mathbb{R}_{+}^{n}$, to the whole of $\mathbb{R}_{+}^{n}$. Thus

$$
\mathbb{R}_{+}^{n}=\left\{(x, y): x \in \mathbb{R}^{n-1}, y>0\right\}, \quad \text { and } \quad \mathbb{R}^{n-1}=\mathbb{R}^{n-1} \times\{0\}=\left\{(x, 0): x \in \mathbb{R}^{n-1}\right\} .
$$

In addition we denote by $\mathcal{B}^{n-1}$ the unit ball of $\mathbb{R}^{n-1}$,

$$
\mathcal{B}^{n-1}=\left\{x \in \mathbb{R}^{n-1}:|x| \leq 1\right\}
$$

and consider a convolution kernel $\mathcal{K} \in C_{c}^{\infty}\left(\mathcal{B}^{n-1}\right)$ such that

$$
\int_{\mathbb{R}^{n-1}} \mathcal{K} d \mathcal{H}^{n-1}=1 .
$$

Put, for $x \in \mathbb{R}^{n-1}$ and $y>0$,

$$
\mathcal{K}_{y}(x)=y^{1-n} \mathcal{K}\left(\frac{x}{y}\right)
$$

and define the operator

$$
\mathcal{E}^{+}: f(x) \mapsto F(x, y)=\left(\mathcal{K}_{y} * f\right)(x),
$$

where $*$ denotes convolution of functions on $\mathbb{R}^{n-1}$ (defined component-wise when $f$ is vector-valued). Let us recall the definition of the homogeneous Sobolev space $\mathcal{W}^{1, p}=\mathcal{W}^{1, p}\left(\mathbb{R}_{+}^{n}, \mathbb{R}^{N}\right)$ for $1 \leq p<\infty$ as the space of locally integrable maps $v: \mathbb{R}_{+}^{n} \rightarrow \mathbb{R}^{N}$ such that $D v \in L^{p}\left(\mathbb{R}_{+}^{n}, \mathbb{R}^{N \times n}\right)$. Our main result is the following:

Proposition 1.1. The linear extension operator $\mathcal{E}^{+}$defined by (1.1) maps $W^{1, p}\left(\mathbb{R}^{n-1}, \mathbb{R}^{N}\right)$ continuously into $\mathcal{W}^{1, p}\left(\mathbb{R}_{+}^{n}, \mathbb{R}^{N}\right), p \geq 1$. Moreover we have for $p \in(1, \infty)$ :

i) If $f \in W^{1, p}\left(\mathbb{R}^{n-1}, \mathbb{R}^{N}\right) \cap L^{r}\left(\mathbb{R}^{n-1}, \mathbb{R}^{N}\right)$ and $q=p+\frac{r}{n-1+r}$, then there exists a positive constant $c$ depending only on $n, N, r, p$ such that

$$
\|F\|_{L^{q^{\star}}\left(\mathbb{R}_{+}^{n}, \mathbb{R}^{N}\right)}^{q}+\|D F\|_{L^{q}\left(\mathbb{R}_{+}^{n}, \mathbb{R}^{N \times n}\right)}^{q} \leq c\|D f\|_{L^{p}\left(\mathbb{R}^{n-1}, \mathbb{R}^{N \times(n-1)}\right)}^{p}\|f\|_{L^{r}\left(\mathbb{R}^{n-1}, \mathbb{R}^{N}\right)}^{\frac{r}{n+r}} .
$$


ii) If $f \in W^{1, p}\left(\mathbb{R}^{n-1}, \mathbb{R}^{N}\right) \cap B M O\left(\mathbb{R}^{n-1}, \mathbb{R}^{N}\right)$ and $q=p+1$, then there exists a positive constant $c$ depending only on $n, N$, $p$ such that

$$
\|F\|_{L^{q^{\star}\left(\mathbb{R}_{+}^{n}, \mathbb{R}^{N}\right)}}^{q}+\|D F\|_{L^{q}\left(\mathbb{R}_{+}^{n}, \mathbb{R}^{N \times n}\right)}^{q} \leq c\|D f\|_{L^{p}\left(\mathbb{R}^{n-1}, \mathbb{R}^{N \times(n-1)}\right)}^{p}\|f\|_{B M O\left(\mathbb{R}^{n-1}, \mathbb{R}^{N}\right)} \cdot
$$

iii) If $f \in W^{1, p}\left(\mathbb{R}^{n-1}, \mathbb{R}^{N}\right) \cap C^{0, \alpha}\left(\mathbb{R}^{n-1} ; \mathbb{R}^{N}\right)$ and $q=p+\frac{1}{1-\alpha}$ then there exists a positive constant c depending only on $n, N, \alpha, p$ such that

$$
\|F\|_{L^{q^{\star}\left(\mathbb{R}_{+}^{n}, \mathbb{R}^{N}\right)}}^{q}+\|D F\|_{L^{q}\left(\mathbb{R}_{+}^{n}, \mathbb{R}^{N \times n}\right)}^{q} \leq c\|D f\|_{L^{p}\left(\mathbb{R}^{n-1}, \mathbb{R}^{N \times(n-1)}\right)}^{p}\|f\|_{C^{0, \alpha}\left(\mathbb{R}^{n-1}, \mathbb{R}^{N}\right)}^{\frac{1}{1-\alpha}},
$$

where as usual $q^{\star}=\frac{n q}{n-q}$ denotes the Sobolev conjugate exponent of $q$ if $q<n$, or any finite exponent if $q \geq n$.

We transfer this construction to functions defined on spheres in $\mathbb{R}^{n}$, as usual by the use of the stereographic projections. This will allow us to connect two $W^{1, p}$ functions in an annulus with a $W^{1, q}$ function, for some $q \geq p$. It is well known that, when dealing with integral functionals with $(p, q)$-growth conditions, one of the main difficulties is to construct suitable test functions and this is the motivation for our result.

Here, as an application, we will establish the $C^{1, \alpha}$ partial regularity of the local minimizers of quasiconvex integrals of the form

$$
\mathcal{I}(v, O)=\int_{O} G(D v(x)) \mathrm{d} x
$$

where $O$ is an open subset of some fixed bounded and open subset $\Omega$ of $\mathbb{R}^{n}, n>2$, and $v: \Omega \rightarrow \mathbb{R}^{N}, N \geq 2$. We consider integrands $G: \mathbb{R}^{N \times n} \rightarrow \mathbb{R}$ satisfying for some positive constants $L, \nu>0$ the following hypotheses:

$$
\begin{gathered}
G: \mathbb{R}^{N \times n} \rightarrow \mathbb{R} \text { is } C^{2} \\
0 \leq G(\xi) \leq L\left(1+|\xi|^{n}\right) \\
\int_{\Omega} G(\xi+D \varphi(x)) \mathrm{d} x \geq \int_{\Omega}\left(G(\xi)+\nu\left(1+|D \varphi(x)|^{2}\right)^{\frac{n-3}{2}}|D \varphi(x)|^{2}\right) \mathrm{d} x
\end{gathered}
$$

for every $\xi \in \mathbb{R}^{N \times n}$ and for all $\varphi \in W_{0}^{1, n-1}\left(\Omega, \mathbb{R}^{N}\right)$. An example of a functional that is not convex and satisfies the above assumptions in dimensions $n=N>2$ is given by

$$
\int_{O}\left(1+|D u|^{2}\right)^{\frac{n-1}{2}}+|\operatorname{Adj} D u|^{\frac{n}{n-1}} .
$$

The quasiconvexity condition (H3) is a uniform strict form of the $W^{1, n-1}$-quasiconvexity condition considered in [3]. See also [32].

The notion of quasiconvexity, originally introduced in [42] as a condition, that under suitable growth conditions, is equivalent to sequential weak lower semicontinuity, has nowadays become a common condition in the vectorial calculus of variations.

The study of regularity of minimizers of quasiconvex integrals started with the celebrated paper by Evans [20] and has subsequently been studied by many authors (see for instance [28, 1, 11, 7, 12, 13, 30, 17] for results under standard growth condition). We recall that for vector valued minimizers the regularity results are only partial, meaning that they are obtained outside a negligible relatively closed subset of $\Omega$. This state of affairs is even unavoidable for the case of regular variational problems (i.e. strongly convex integrands) by well-known examples, see for instance [15, 43, 48, 41].

The assumptions (H1) - (H3) clearly entail a $(p, q)$ growth condition with $p=n-1$ and $q=n$. The study of the regularity of minimizers of such functionals started with the celebrated papers by Marcellini (see in particular $[36,38])$ and has since attracted much attention. From very early on it has been clear that no regularity can be expected if the coercivity and growth exponents, denoted $p$ and $q$, respectively, are too far apart (see [27, 37]). On the other hand, many regularity results are available for convex integrands if the ratio $q / p$ is bounded above by a suitable constant depending on the dimension $n$, and converging to 1 when $n$ tends to infinity (incl.[2, 4, 5, 6, 8, $16,18,19,21,34,39,40,44])$. 
The first papers regarding the non convex $(p, q)$ growth case are due to Fusco and Hutchinson ([24, 25]). More precisely, they considered polyconvex integral functionals satisfying a $(p, q)$ growth condition of the type $n-1<p<q=n$. We emphasize that their techniques can not be used to treat the general quasiconvex case.

More recently, the general quasiconvex case has been addressed in a series of papers by Schmidt $([45,46,47])$ under a $(p, q)$ growth assumption of the type $p<q<p+\min \left\{\frac{1}{n}, \frac{p}{2 n}\right\}$. Here, as an application of the trace preserving operator, we give an extension of one of the results by Schmidt ([45]) to the case $p=n-1, q=n$ and, in this way, we cover what appears to be a borderline case. At least this is so for the corresponding situation in the Lebesgue-Serring-Marcelini set-up, where the functional is defined by relaxation from smooth maps (see the example of Malý [35]).

Definition 1.1. A map $u \in W_{\text {loc }}^{1, n-1}\left(\Omega, \mathbb{R}^{N}\right)$ is a local $G$-minimizer if

$$
\int_{O} G(D u) \mathrm{d} x \leq \int_{O} G(D v) \mathrm{d} x
$$

for any $O \Subset \Omega$ and any $v \in W_{u}^{1, n-1}\left(O, \mathbb{R}^{N}\right)$.

Recall that quasiconvexity in connection with the growth condition (H2) implies

$$
|D G(\xi)| \leq c\left(1+|\xi|^{n-1}\right) \text {. }
$$

(for the proof see [36], Step 2, p. 6).

Moreover, the strict uniform quasiconvexity condition, stated in (H3), implies the strong Legendre-Hadamard condition (see [42]), that is:

$$
D^{2} G(A)(b \otimes a, b \otimes a) \geq \gamma|b|^{2}|a|^{2}
$$

for all $b \in \mathbb{R}^{N}, a \in \mathbb{R}^{n}$, where as usual $b \otimes a$ is the $N \times n$ matrix with entry $b^{r} a^{c}$ in row $r$, column $c$.

Remark 1.1. [The Euler-Lagrange system for $q \leq p+1$.$] If u$ is a local minimizer of the functional $\mathcal{I}$ and $\phi \in C_{c}^{1}\left(\Omega, \mathbb{R}^{N}\right)$ we get by use of (H4) and the minimality condition that for any $\varepsilon>0$ :

$$
0 \leq \int_{\Omega}[G(D u+\varepsilon D \phi)-G(D u)] \mathrm{d} x=\varepsilon \int_{\Omega} \int_{0}^{1} \frac{\partial G}{\partial \xi_{\alpha}^{i}}(D u+\varepsilon t D \phi) D_{\alpha} \phi^{i} \mathrm{~d} t \mathrm{~d} x,
$$

where the usual summation convention is in force. Dividing this inequality by $\varepsilon$, and letting $\varepsilon \searrow 0$, we infer from (H4) since $q \leq p+1$, that

$$
\int_{\Omega} \frac{\partial G}{\partial \xi_{\alpha}^{i}}(D u) D_{\alpha} \phi^{i} \mathrm{~d} x \geq 0
$$

Consequently, $u$ is a weak solution to the Euler-Lagrange system for $\mathcal{I}$ :

$$
\int_{\Omega} \frac{\partial G}{\partial \xi_{\alpha}^{i}}(D u) D_{\alpha} \phi^{i} \mathrm{~d} x=0 \quad \forall \phi \in C_{c}^{1}\left(\Omega, \mathbb{R}^{N}\right) .
$$

We recall that the connection between $G$-extremality and $G$-minimality has been considered in [9], [10] for convex integrands, under more general assumptions on the growth conditions of $G$. Actually in these papers it has been proven that minimizers satisfy the Euler-Lagrange system assuming that the convex integrand is only superlinear at $\infty$ without any growth assumption from above.

Notice that we do not assume any growth condition on the second derivatives of $G$ and that they do not follow from our hypotheses (H1)-(H3). Instead we observe that since $G$ is $C^{2}$ we have for each $M>0$ the bound

$$
\sup \left\{\left|D^{2} G(A)\right|:|A| \leq M\right\}=: K_{M}<\infty .
$$

We will prove the following $C^{1, \alpha}$ - partial regularity result for local $G$-minimizers.

Theorem 1.1. Let $G$ satisfy the assumptions (H1), (H2), (H3). If $u \in W_{\mathrm{loc}}^{1, n-1}\left(\Omega, \mathbb{R}^{N}\right)$ is a local G-minimizer, then there exists an open subset $\Omega_{0}$ of $\Omega$ such that

$$
\operatorname{meas}\left(\Omega \backslash \Omega_{0}\right)=0
$$


and

$$
u \in C_{\mathrm{loc}}^{1, \alpha}\left(\Omega_{0}, \mathbb{R}^{N}\right) \quad \text { for all } \quad \alpha<1 .
$$

The second application of the trace preserving operator concerns integral bounds for weak solutions to a nonlinear elliptic system and is presented in Section 4.

Acknowledgement: M. Carozza and A. Passarelli di Napoli are members of the Gruppo Nazionale per l'Analisi Matematica, la Probabilità e le loro Applicazioni (GNAMPA) of the Istituto Nazionale di Alta Matematica (INdAM).

\section{AN EXTENSION OPERATOR}

The aim of this section is to give the proof of our main result from which we will deduce that two $W^{1, p}$ functions can be connected in an annulus with a $W^{1, q}$ function, for some $q>p$ (see Lemmas 2.1 and 2.2 below).

Proof of Proposition 1.1. Because $F(x, y) \rightarrow 0$ uniformly in $x \in \mathbb{R}^{n-1}$ as $y \rightarrow \infty$, it follows from the PoincaréSobolev inequality (when $q<n$ ) that

$$
\|F\|_{L^{q^{\star}\left(\mathbb{R}_{+}^{n}, \mathbb{R}^{N}\right)}}^{q} \leq c(n, N, q)\|D F\|_{L^{q}\left(\mathbb{R}_{+}^{n}, \mathbb{R}^{N \times n}\right)}^{q} .
$$

Obviously, when $q=n$ we will have a BMO bound and when $q>n$ a $\left(1-\frac{n}{q}\right)$-Hölder bound. Hence we can confine ourselves to estimating only the derivatives of $F$. Carrying out the differentiation gives

$$
\begin{aligned}
D_{x_{i}} F(x, y) & =\left(\mathcal{K}_{y} * D_{x_{i}} f\right)(x) \\
& =\frac{1}{y}\left(\left(D_{x_{i}} \mathcal{K}\right)_{y} * f\right)(x),
\end{aligned}
$$

where clearly $D_{x_{i}} \mathcal{K} \in C_{c}^{\infty}\left(\mathcal{B}^{n-1}\right)$ and $\int_{\mathbb{R}^{n-1}} D_{x_{i}} \mathcal{K} d \mathcal{H}^{n-1}=0$. Likewise we may write

$$
D_{y} F(x, y)=\frac{1}{y}\left(\tilde{\mathcal{K}}_{y} * f\right)(x) \text { with } \tilde{\mathcal{K}}=-(n-1) K(x)-D K(x) . x .
$$

Note that $\tilde{\mathcal{K}} \in C_{c}^{\infty}\left(\mathcal{B}^{n-1}\right)$ and $\int_{\mathbb{R}^{n-1}} \tilde{\mathcal{K}} d \mathcal{H}^{n-1}=0$. Moreover, the definition of $F$ and a simple change of variables yield

$$
D_{y} F(x, y)=-\int_{\mathbb{R}^{n-1}} \mathcal{K}(z)\langle D f(x-y z), z\rangle d \mathcal{H}^{n-1}(z) .
$$

First we observe that one easily gets that the operator $\mathcal{E}^{+}$maps $W^{1, p}\left(\mathbb{R}^{n-1}, \mathbb{R}^{N}\right)$ continuously into $\mathcal{W}^{1, p}\left(\mathbb{R}_{+}^{n}, \mathbb{R}^{N}\right)$. Namely, for each fixed $x \in \mathbb{R}^{n-1}$, we have

$$
\begin{aligned}
\int_{0}^{\infty}\left|D_{y} F(x, y)\right|^{p} \mathrm{~d} y= & \int_{0}^{1}\left|\int_{\mathbb{R}^{n-1}} \mathcal{K}(z)\langle D f(x-y z), z\rangle d \mathcal{H}^{n-1}(z)\right|^{p} \mathrm{~d} y \\
& +\int_{1}^{\infty} \mid \int_{\mathbb{R}^{n-1}} \frac{1}{y}\left(\left.\tilde{\mathcal{K}}_{y}(z) f(x-z) d \mathcal{H}^{n-1}(z)\right|^{p} \mathrm{~d} y\right. \\
\leq & c(n)\|\mathcal{K}\|_{L^{\infty}}^{p} M(|D f|)^{p}(x)+c(n)\|\tilde{\mathcal{K}}\|_{L^{\infty}}^{p} M(|f|)^{p}(x) .
\end{aligned}
$$

Here and in the following we denote by $M(g)$ the Hardy-Littlewood maximal function of $g$. Integrating (2.4) over $x \in \mathbb{R}^{n-1}$ and using the maximal theorem we obtain

$$
\int_{\mathbb{R}_{+}^{n}}\left|D_{y} F(x, y)\right|^{p} \mathrm{~d} x \mathrm{~d} y \leq c(n, N, p) \int_{\mathbb{R}^{n-1}}\left(|D f(x)|^{p}+|f(x)|^{p}\right) \mathrm{d} x .
$$

Similar bounds hold for the other partial derivatives. Next step is to prove inequalities (1.2)-(1.4). To this aim we prove a corresponding inequality for each fixed $x \in \mathbb{R}^{n-1}$ and then we integrate the result with respect to $x$. 
We will examine only the $y$-derivative of $F$, since the computations for the other partial derivatives are analogous. First write

$$
\begin{aligned}
\int_{0}^{\infty}\left|D_{y} F(x, y)\right|^{q} \mathrm{~d} y= & \int_{0}^{\delta}\left|\int_{\mathbb{R}^{n-1}} \mathcal{K}(z)\langle D f(x-y z), z\rangle d \mathcal{H}^{n-1}(z)\right|^{q} \mathrm{~d} y \\
& +\int_{\delta}^{\infty}\left|\int_{\mathbb{R}^{n-1}} \frac{1}{y} \tilde{\mathcal{K}}_{y}(z) f(x-z) d \mathcal{H}^{n-1}(z)\right|^{q} \mathrm{~d} y \\
=: & I+I I .
\end{aligned}
$$

In order to estimate the term $I$ it suffices to observe that

$$
\begin{aligned}
I & \leq \int_{0}^{\delta}\left(\int_{\mathcal{B}^{n-1}}|\mathcal{K}(z)||D f(x-y z)| d \mathcal{H}^{n-1}(z)\right)^{q} \mathrm{~d} y \\
& \leq c(n)\|\mathcal{K}\|_{L^{\infty}}^{q} M(|D f|)^{q}(x) \delta
\end{aligned}
$$

where $M(D f)$ is the (Hardy-Littlewood) maximal function of $D f$. We shall estimate the term $I I$ in three separate cases.

Case 1: $f \in W^{1, p}\left(\mathbb{R}^{n-1}, \mathbb{R}^{N}\right) \cap L^{r}\left(\mathbb{R}^{n-1}, \mathbb{R}^{N}\right)$.

Thanks to Hölder's inequality and the assumptions on $\tilde{\mathcal{K}}$ and $f$, we have that

$$
\begin{aligned}
I I & \leq\|f\|_{L^{r}}^{q} \int_{\delta}^{\infty} y^{-n q}\left(\int_{\mathbb{R}^{n-1}} \tilde{\mathcal{K}}^{\frac{r}{r-1}}\left(\frac{z}{y}\right) d \mathcal{H}^{n-1}(z)\right)^{q \frac{r-1}{r}} \mathrm{~d} y \\
& \leq c(n)\|\tilde{\mathcal{K}}\|_{L^{\infty}}^{q}\|f\|_{L^{r}}^{q} \int_{\delta}^{\infty} y^{-n q} y^{(n-1) q \frac{r-1}{r}} \mathrm{~d} y \\
& \leq c(n, q, r)\|\tilde{\mathcal{K}}\|_{L^{\infty}}^{q}\|f\|_{L^{r}}^{q} \delta^{-n q+1+(n-1) q \frac{r-1}{r}}
\end{aligned}
$$

where we used that, since the support of $\tilde{\mathcal{K}}$ is contained in the unit ball of $\mathbb{R}^{n-1}$, then

$$
\int_{\mathbb{R}^{n-1}} \tilde{\mathcal{K}}^{\frac{r}{r-1}}\left(\frac{z}{y}\right) d \mathcal{H}^{n-1}(z) \leq c(n)\|\tilde{\mathcal{K}}\|_{L^{\infty}} y^{n-1},
$$

and also that, by virtue of the assumption on the exponent $q$, we have that $-n q+1+(n-1) q \frac{r-1}{r}<0$. Now, if we choose

in (2.7) and in (2.8), we obtain that

$$
\delta=\left(\frac{\|f\|_{L^{r}}}{M(|D f|)(x)}\right)^{\frac{r}{n-1+r}}
$$

$$
I+I I=\int_{0}^{\infty}\left|D_{y} F(x, y)\right|^{q} \mathrm{~d} y \leq c\left(n, q, r,\|\tilde{\mathcal{K}}\|_{L^{\infty}}\right)\|f\|_{L^{r}}^{\frac{r}{n-1+r}} M(|D f|)^{q-\frac{r}{n-1+r}}(x)
$$

Integrating (2.9) with respect to $x \in \mathbb{R}^{n-1}$, we get

$$
\int_{\mathbb{R}_{+}^{n}}\left|D_{y} F(x, y)\right|^{q} \mathrm{~d} x \mathrm{~d} y \leq c\left(n, N, q, r,\|\tilde{\mathcal{K}}\|_{L^{\infty}}\right)\|f\|_{L^{r}}^{\frac{r}{n-1+r}} \int_{\mathbb{R}^{n-1}}|D f|^{p}(x) \mathrm{d} x
$$

provided $q=p+\frac{r}{n-1+r}$, by virtue of the maximal theorem.

Case 2: $f \in W^{1, p}\left(\mathbb{R}^{n-1}, \mathbb{R}^{N}\right) \cap B M O\left(\mathbb{R}^{n-1}, \mathbb{R}^{N}\right)$.

Denoting by $f_{B}$ the mean value of $f$ on the ball $B(0,|y|)$, we have that

$$
\begin{aligned}
I I & =\int_{\delta}^{\infty} \mid \int_{\mathbb{R}^{n-1}} \frac{1}{y}\left(\left.\tilde{\mathcal{K}}_{y}(z)\left(f(x-z)-f_{B}\right) d \mathcal{H}^{n-1}(z)\right|^{q} \mathrm{~d} y\right. \\
& \leq c(n)\|\tilde{\mathcal{K}}\|_{L^{\infty}}^{q} \int_{\delta}^{\infty} \frac{1}{y^{q}}\left(f_{B(0,|y|)}\left|f(x-z)-f_{B}\right| d \mathcal{H}^{n-1}(z)\right)^{q} \mathrm{~d} y
\end{aligned}
$$




$$
\begin{aligned}
& \leq c(n)\|\tilde{\mathcal{K}}\|_{L^{\infty}}^{q}\|f\|_{B M O}^{q} \int_{\delta}^{\infty} y^{-q} \mathrm{~d} y \\
& \leq c(n, q)\|\tilde{\mathcal{K}}\|_{L^{\infty}}^{q}\|f\|_{B M O}^{q} \delta^{1-q} .
\end{aligned}
$$

Then, inserting (2.7) and (2.11) in (2.6), we get

$$
\begin{aligned}
\int_{0}^{\infty}\left|D_{y} F(x, y)\right|^{q} \mathrm{~d} y \leq & c(n)\|\mathcal{K}\|_{L^{\infty}}^{q} M(|D f|)^{q}(x) \delta \\
& +c(n, q)\|\tilde{\mathcal{K}}\|_{L^{\infty}}^{q}\|f\|_{B M O}^{q} \delta^{1-q} .
\end{aligned}
$$

It is easy to verify that for

the following equality

$$
\delta=\|f\|_{B M O} M(|D f|)^{-1}(x)
$$

$$
M(|D f|)^{q}(x) \delta=\|f\|_{B M O}^{q} \delta^{1-q}
$$

is satisfied. Then inserting such $\delta$ in (2.12) we obtain

$$
\int_{0}^{\infty}\left|D_{y} F(x, y)\right|^{q} \mathrm{~d} y \leq c\left(n, q,\|\tilde{\mathcal{K}}\|_{L^{\infty}}\right)\|f\|_{B M O} M(|D f|)^{q-1}(x) .
$$

Now, integrating both sides of (2.13) with respect to $x \in \mathbb{R}^{n-1}$ and using the maximal theorem, we get

$$
\begin{aligned}
\int_{\mathbb{R}_{+}^{n}}\left|D_{y} F(x, y)\right|^{q} \mathrm{~d} x \mathrm{~d} y & \leq c(n, q)\|\mathcal{K}\|_{L^{\infty}}^{q}\|f\|_{B M O} \int_{\mathbb{R}^{n-1}} M(|D f|)^{q-1}(x) \mathrm{d} x \\
& \leq c(n, N, q)\|\mathcal{K}\|_{L^{\infty}}^{q}\|f\|_{B M O} \int_{\mathbb{R}^{n-1}}|D f|^{p}(x) \mathrm{d} x,
\end{aligned}
$$

provided $q=p+1$.

Case 3: $f \in W^{1, p}\left(\mathbb{R}^{n-1}, \mathbb{R}^{N}\right) \cap C^{0, \alpha}\left(\mathbb{R}^{n-1}, \mathbb{R}^{N}\right)$.

Thanks to our assumptions on $\mathcal{K}$, we have that

$$
\begin{aligned}
I I & =\int_{\delta}^{\infty} \mid \int_{\mathbb{R}^{n-1}} \frac{1}{y}\left(\left.\tilde{\mathcal{K}}_{y}(z)(f(x-z)-f(x)) d \mathcal{H}^{n-1}(z)\right|^{q} \mathrm{~d} y\right. \\
& \leq\|f\|_{C^{0, \alpha}}^{q} \int_{\delta}^{\infty}\left(\int_{\mathbb{R}^{n-1}}\left|\tilde{\mathcal{K}}_{y}(z)\right| d \mathcal{H}^{n-1}(z)\right)^{q} y^{(\alpha-1) q} \mathrm{~d} y \\
& \leq c(\alpha, n, q)\|\tilde{\mathcal{K}}\|_{L^{\infty}}^{q}\|f\|_{C^{0, \alpha}}^{q} \delta^{1+(\alpha-1) q} .
\end{aligned}
$$

Then, inserting (2.7) and (2.15) in (2.6), we get

$$
\begin{aligned}
\int_{0}^{\infty}\left|D_{y} F(x, y)\right|^{q} \mathrm{~d} y \leq & c(n)\|\mathcal{K}\|_{L^{\infty}}^{q} M(|D f|)^{q}(x) \delta \\
& +c(\alpha, n, q)\|\tilde{\mathcal{K}}\|_{L^{\infty}}^{q}\|f\|_{C^{0, \alpha}}^{q} \delta^{1+(\alpha-1) q} .
\end{aligned}
$$

If we choose

then

$$
\delta=M(|D f|)^{\frac{1}{\alpha-1}}(x)\|f\|_{C^{0, \alpha}}^{\frac{1}{1-\alpha}}
$$

Thus inserting such $\delta$ in (2.16) we obtain

$$
M(|D f|)^{q}(x) \delta=\|f\|_{C^{0, \alpha}}^{q} \delta^{1+(\alpha-1) q} .
$$

$$
\int_{0}^{\infty}\left|D_{y} F(x, y)\right|^{q} \mathrm{~d} y \leq c\left(\alpha, n, q,\|\tilde{\mathcal{K}}\|_{L^{\infty}}\right)\|f\|_{C^{0, \alpha}}^{\frac{1}{1-\alpha}} M(|D f|)^{q+\frac{1}{\alpha-1}}(x)
$$

Now, integrating both sides of (2.17) with respect to $x \in \mathbb{R}^{n-1}$ and using the maximal theorem, we get

$$
\int_{\mathbb{R}_{+}^{n}}\left|D_{y} F(x, y)\right|^{q} \mathrm{~d} x \mathrm{~d} y \leq c\left(\alpha, n, q,\|\tilde{\mathcal{K}}\|_{L^{\infty}}\right)\|f\|_{C^{0, \alpha}}^{\frac{1}{1-\alpha}} \int_{\mathbb{R}^{n-1}} M(|D f|)^{q+\frac{1}{\alpha-1}}(x) \mathrm{d} x
$$




$$
\leq c\left(\alpha, n, N, q,\|\tilde{\mathcal{K}}\|_{L^{\infty}}\right)\|f\|_{C^{0, \alpha}}^{\frac{1}{1-\alpha}} \int_{\mathbb{R}^{n-1}}|D f|^{p}(x) \mathrm{d} x,
$$

provided $q=p+\frac{1}{1-\alpha}$.

In order to conclude the proof it is enough to observe that estimates (2.10), (2.14), and (2.18) hold true for all the derivatives of $F(x, y)$, then summing up we conclude with (1.2), (1.3), and (1.4), respectively.

Remark 2.1. Observe that letting $r \nearrow \infty$ in (1.2) we obtain an estimate analogous to (1.3), where $\|f\|_{B M O}$ is replaced by $\|f\|_{L^{\infty}}$. Moreover, if the function $f \in W^{1, p}\left(\mathbb{R}^{n-1}, \mathbb{R}^{N}\right)$ for a $p<n-1$, but does not satisfy any other regularity assumption, then by the Sobolev embedding theorem we know that $f \in L^{r}\left(\mathbb{R}^{n-1}, \mathbb{R}^{N}\right)$, with $r=\frac{(n-1) p}{n-1-p}$. In this case the exponent of improved integrability is given by $q=\frac{n p}{n-1}$, which is exactly the borderline case determined by Lemma 2.4 in [22]. However, note that our result holds true also for the borderline exponent $q=\frac{n p}{n-1}$, whereas for the result in [22] the exponent $q$ has to be strictly less than $\frac{n p}{n-1}$. The borderline case was also discussed in [31].

We also note that if $f \in W^{1, n-1}\left(\mathbb{R}^{n-1}, \mathbb{R}^{N}\right)$, then Sobolev's embedding theorem implies that $f \in B M O\left(\mathbb{R}^{n-1}, \mathbb{R}^{N}\right)$. Hence any map $f \in W^{1, n-1}\left(\mathbb{R}^{n-1}, \mathbb{R}^{N}\right)$ can be extended to a map $F$ belonging to $\mathcal{W}^{1, n}\left(\mathbb{R}_{+}^{n}, \mathbb{R}^{N}\right)$ and

$$
\|D F\|_{L^{n}\left(\mathbb{R}_{+}^{n}, \mathbb{R}^{N \times n}\right)}^{n} \leq C(n, N)\|D f\|_{L^{n-1}\left(\mathbb{R}^{n-1}, \mathbb{R}^{N \times(n-1)}\right)}^{n} .
$$

For further needs we now prove the following

Proposition 2.1. The linear extension operator $\mathcal{E}^{+}$defined by (1.1) maps $W^{1,2}\left(\mathbb{R}^{n-1}, \mathbb{R}^{N}\right)$ continuously into $\mathcal{W}^{1,2}\left(\mathbb{R}_{+}^{n}, \mathbb{R}^{N}\right)$. Moreover we have that there exists a positive constant $c$ depending on $n, N$ such that

$$
\|D F\|_{L^{2}\left(\mathbb{R}_{+}^{n}, \mathbb{R}^{N \times n}\right)}^{2} \leq c\|D f\|_{L^{2}\left(\mathbb{R}^{n-1}, \mathbb{R}^{N \times(n-1)}\right)}^{2} .
$$

Proof. By the definition of $F(x, y)$ and by virtue of the equality (2.2) we have that

$$
\begin{aligned}
\int_{\mathbb{R}_{+}^{n}}\left|D_{y} F\right|^{2} \mathrm{~d} x \mathrm{~d} y & =\int_{0}^{+\infty} \int_{\mathbb{R}^{n-1}}\left|\tilde{\mathcal{K}}_{y} \star f\right|^{2} \frac{\mathrm{d} y}{y^{2}} \mathrm{~d} x \\
& =\int_{0}^{+\infty} \int_{\mathbb{R}^{n-1}}|\widehat{\tilde{\mathcal{K}}} y|^{2}|\widehat{f}|^{2} \frac{\mathrm{d} y}{y^{2}} \mathrm{~d} x \\
& =\int_{0}^{+\infty} \int_{\mathbb{R}^{n-1}}|\widehat{\tilde{\mathcal{K}}}(y \xi)|^{2}|\widehat{f}(\xi)|^{2} \frac{\mathrm{d} y}{y^{2}} d \xi \\
& \leq c \int_{0}^{\frac{1}{|\xi|}} \int_{\mathbb{R}^{n-1}}|y \xi|^{2}|\widehat{f}(\xi)|^{2} \frac{\mathrm{d} y}{y^{2}} d \xi+c \int_{\frac{1}{|\xi|}}^{+\infty} \int_{\mathbb{R}^{n-1}}|y \xi|^{-2}|\widehat{f}(\xi)|^{2} \frac{\mathrm{d} y}{y^{2}} d \xi \\
& \leq c \int_{\mathbb{R}^{n-1}}|\xi||\widehat{f}(\xi)|^{2} d \xi \leq c\|f\|_{W^{1 / 2,2}\left(\mathbb{R}^{n-1}, \mathbb{R}^{N}\right)} \leq c\|D f\|_{L^{2}\left(\mathbb{R}^{n-1}, \mathbb{R}^{N \times(n-1)}\right)},
\end{aligned}
$$

where the symbol $\widehat{h}$ denotes the Fourier transform of $h$ and we used that

$$
|\widehat{\tilde{\mathcal{K}}}(\eta)| \leq c \min \left\{|\eta|, \frac{1}{|\eta|}\right\} .
$$

It is now routine to construct linear extension operators from smooth hypersurfaces in $\mathbb{R}^{n}$ with similar mapping properties. Here we shall confine ourselves to spheres where the previous construction can be transferred by use of two stereographic projections and a standard covering argument. Our results can be translated into the following

Corollary 2.1. Let $v \in W^{1, p}\left(B_{R}, \mathbb{R}^{N}\right)$, with $p>1$. Then there exists a function $\tilde{v} \in W^{1, p}\left(\mathbb{R}^{n}, \mathbb{R}^{N}\right)$ such that

$$
\tilde{v}=v \quad \text { on } \partial B_{R}
$$

and

j) if $v \in L^{r}\left(\partial B_{R}, \mathbb{R}^{N}\right)$ with $\frac{(n-1) p}{n-1-p} \leq r<+\infty$, then

$$
\|\tilde{v}\|_{L^{q^{\star}}\left(\mathbb{R}^{n} \backslash B_{R}, \mathbb{R}^{N}\right)}^{q}+\|D \tilde{v}\|_{L^{q}\left(\mathbb{R}^{n} \backslash B_{R}, \mathbb{R}^{N \times n}\right)}^{q} \leq c(n, N, p, r)\|D v\|_{L^{p}\left(\partial B_{R}, \mathbb{R}^{N \times(n-1)}\right)}^{p}\|v\|_{L^{r}\left(\partial B_{R}, \mathbb{R}^{N}\right)}^{q-p}
$$


where $q=p+\frac{r}{n-1+r}$.

jj) If $v \in B M O\left(\partial B_{R}, \mathbb{R}^{N}\right)$, then

(2.21) $\quad\|\tilde{v}\|_{L^{q^{\star}}\left(\mathbb{R}^{n} \backslash B_{R}, \mathbb{R}^{N}\right)}^{q}+\|D \tilde{v}\|_{L^{q}\left(\mathbb{R}^{n} \backslash B_{R}, \mathbb{R}^{N \times n}\right)}^{q} \leq c(n, N, p)\|D v\|_{L^{p}\left(\partial B_{R}, \mathbb{R}^{N \times(n-1)}\right)}^{p}\|v\|_{B M O\left(\partial B_{R}, \mathbb{R}^{N}\right)}$, where $q=p+1$.

jjj) If $v \in C^{0, \alpha}\left(\partial B_{R}, \mathbb{R}^{N}\right)$, then

(2.22) $\|\tilde{v}\|_{L^{q^{\star}}\left(\mathbb{R}^{n} \backslash B_{R}, \mathbb{R}^{N}\right)}^{q}+\|D \tilde{v}\|_{L^{q}\left(\mathbb{R}^{n} \backslash B_{R}, \mathbb{R}^{N \times n}\right)}^{q} \leq c(n, N, \alpha, p)\|D v\|_{L^{p}\left(\partial B_{R}, \mathbb{R}^{N \times(n-1)}\right)}^{p}\|v\|_{C^{0}, \alpha}^{\frac{1}{1-\alpha}\left(\partial B_{R}, \mathbb{R}^{N}\right)}$, where $q=p+\frac{1}{1-\alpha}$.

Via the inversion in the sphere we get also

Corollary 2.2. Let $w \in W^{1, p}\left(B_{R}, \mathbb{R}^{N}\right)$ with $p>1$. Then there exists a function $\tilde{w} \in W^{1, p}\left(\mathbb{R}^{n}, \mathbb{R}^{N}\right)$ such that

$$
\tilde{w}=w \quad \text { on } \quad \partial B_{R}
$$

and

h) if $w \in L^{r}\left(\partial B_{R}, \mathbb{R}^{N}\right)$ with $\frac{(n-1) p}{n-1-p} \leq r<+\infty$ then, for every $\gamma \in(0,1)$

$$
\|\tilde{w}\|_{L^{q^{\star}\left(B_{R} \backslash B_{\gamma R}, \mathbb{R}^{N}\right)}}^{q}+\|D \tilde{w}\|_{L^{q}\left(B_{R} \backslash B_{\gamma R}, \mathbb{R}^{N \times n}\right)}^{q} \leq \frac{1}{\gamma^{2}} c(n, N, p, r)\|D w\|_{L^{p}\left(\partial B_{R}, \mathbb{R}^{N \times(n-1)}\right)}^{p}\|w\|_{L^{r}\left(\partial B_{R}, \mathbb{R}^{N}\right)}^{q-p},
$$

where $q=p+\frac{r}{n-1+r}$.

hh) If $w \in B M O\left(\partial B_{R}, \mathbb{R}^{N}\right)$ then, for every $\gamma \in(0,1)$

$$
\|\tilde{w}\|_{L^{q^{\star}\left(B_{R} \backslash B_{\gamma R}, \mathbb{R}^{N}\right)}}^{q}+\|D \tilde{w}\|_{L^{q}\left(B_{R} \backslash B_{\gamma R}, \mathbb{R}^{N \times n}\right)}^{q} \leq \frac{1}{\gamma^{2}} c(n, N, p)\|D w\|_{L^{p}\left(\partial B_{R}, \mathbb{R}^{N \times(n-1)}\right)}^{p}\|w\|_{B M O\left(\partial B_{R}, \mathbb{R}^{N}\right)},
$$

where $q=p+1$.

hhh) If $v \in C^{0, \alpha}\left(\partial B_{R}, \mathbb{R}^{N}\right)$ then, for every $\gamma \in(0,1)$

(2.25)

$\|\tilde{w}\|_{L^{q^{\star}}\left(B_{R} \backslash B_{\gamma R}, \mathbb{R}^{N}\right)}^{q}+\|D \tilde{w}\|_{L^{q}\left(B_{R} \backslash B_{\gamma R}, \mathbb{R}^{N \times n}\right)}^{q} \leq \frac{1}{\gamma^{2}} c(n, N, \alpha, p)\|D w\|_{L^{p}\left(\partial B_{R}, \mathbb{R}^{N \times(n-1)}\right)}^{p}\|w\|_{C^{0, \alpha}\left(\partial B_{R}, \mathbb{R}^{N}\right)}^{\frac{1}{1-\alpha}}$, where $q=p+\frac{1}{1-\alpha}$.

Proof. Let us consider the inversion in the sphere $S_{x_{0}, R}^{n-1}=\partial B_{x_{0}, R}$, i.e., the map defined as

$$
\Pi(x)=x_{0}+R^{2} \frac{x-x_{0}}{\left|x-x_{0}\right|^{2}} \quad x \in \mathbb{R}^{n} \backslash\left\{x_{0}\right\} .
$$

Recall that $\Pi$ is a conformal mapping of the Riemann sphere $\overline{\mathbb{R}}^{n}$ onto itself. Furthermore

$$
\left|\Pi(x)-x_{0}\right|=\frac{R^{2}}{\left|x-x_{0}\right|}
$$

and

$$
|\Pi(x)-\Pi(y)|=\frac{R^{2}|x-y|}{\left|x-x_{0}\right|\left|y-x_{0}\right|} .
$$

Thanks to (2.27), $\Pi$ is a bilipschitzian mapping of any ring domain $B_{R} \backslash B_{\gamma R}(0<\gamma<1)$ onto itself and the Lipschitz constant is $\frac{1}{\gamma^{2}}$. At this point it suffices to set

$$
\tilde{w}(y)=\bar{w}(\Pi(y)),
$$


where $\bar{w}$ is the function determined by Corollary 2.1 corresponding to $w$ and observe that

$$
|D \tilde{w}| \leq \frac{C}{\gamma^{2}}|D \bar{w}|
$$

Now, if we combine estimates (2.20) and (2.23) in Corollary 2.1 and 2.2 respectively, we can prove the following

Lemma 2.1. Let $v, w \in W^{1, p}\left(B_{1}(0), \mathbb{R}^{N}\right) \cap L^{r}\left(B_{1}(0), \mathbb{R}^{N}\right), p>1$ and $0<s<t<1$. If $p<q \leq p+1$, there exist a function $z \in W^{1, p}\left(B_{1}(0), \mathbb{R}^{N}\right)$ and numbers $0<s<s^{\prime}<t^{\prime}<t<1$, depending on $v$, $w$ such that

$$
\frac{t-s}{8} \leq t^{\prime}-s^{\prime} \leq t-s
$$

and

Moreover

$$
z= \begin{cases}v & \text { on } B_{s^{\prime}} \\ w & \text { on } B_{1} \backslash B_{t^{\prime}}\end{cases}
$$

$$
\int_{B_{t^{\prime}} \backslash B_{s^{\prime}}}|D z|^{q} \mathrm{~d} x \leq C \frac{t^{2}\|v\|_{L^{r}\left(\partial B_{s^{\prime}}, \mathbb{R}^{N}\right)}^{q-p}}{s^{2}(t-s)^{1+\frac{q(n-1)}{n}}}\left(\int_{B_{t} \backslash B_{s}}|D v|^{p} \mathrm{~d} x\right)+C \frac{t^{2}\|w\|_{L^{r}\left(\partial B_{s^{\prime}}, \mathbb{R}^{N}\right)}^{q-p}}{s^{2}(t-s)^{1+\frac{q(n-1)}{n}}}\left(\int_{B_{t} \backslash B_{s}}|D w|^{p} \mathrm{~d} x\right)
$$

and

$$
\left(\int_{B_{t^{\prime}} \backslash B_{s^{\prime}}}|z|^{q^{*}} \mathrm{~d} x\right)^{\frac{q}{q^{*}}} \leq C \frac{t^{2}\|v\|_{L^{r}\left(\partial B_{s^{\prime}}\right)}^{q-p}}{s^{2}(t-s)}\left(\int_{B_{t} \backslash B_{s}}|D v|^{p} \mathrm{~d} x\right)+C \frac{t^{2}\|w\|_{L^{r}\left(\partial B_{s^{\prime}}\right)}^{q-p}}{s^{2}(t-s)}\left(\int_{B_{t} \backslash B_{s}}|D w|^{p} \mathrm{~d} x\right)
$$

where $C=C(n, N, q, p)$ and $q=p+\frac{r}{n-1+r}$.

Proof. Fix $0<s<t<1$ and define the set

$$
\begin{aligned}
E_{s, t}=\left\{\rho \in(s, t): \int_{\partial B_{\rho}}|D v(x)|^{p} d \mathcal{H}^{n-1}(x)\right. & \leq \frac{4}{t-s} \int_{B_{t} \backslash B_{s}}|D v(x)|^{p} \mathrm{~d} x \\
\text { and } \int_{\partial B_{\rho}}|D w(x)|^{p} d \mathcal{H}^{n-1}(x) & \left.\leq \frac{4}{t-s} \int_{B_{t} \backslash B_{s}}|D w(x)|^{p} \mathrm{~d} x\right\} .
\end{aligned}
$$

One can easily check that $\left|E_{s, t}\right| \geq \frac{t-s}{2}$ and therefore we can fix radii $s^{\prime}, t^{\prime} \in E_{s, t}$ such that $s^{\prime}<t^{\prime}$ and $t^{\prime}-s^{\prime}>$ $\frac{t-s}{8}$. Using Corollary 2.1 there exists a function $\tilde{v} \in W^{1, p}\left(\mathbb{R}^{n}, \mathbb{R}^{N}\right)$ such that

$$
\tilde{v}=v \quad \text { on } \quad \partial B_{s^{\prime}}
$$

and

$$
\|\tilde{v}\|_{L^{q^{\star}}\left(\mathbb{R}^{n} \backslash B_{s^{\prime}}, \mathbb{R}^{N}\right)}^{q}+\|D \tilde{v}\|_{L^{q}\left(\mathbb{R}^{n} \backslash B_{s^{\prime}}, \mathbb{R}^{N \times n}\right)}^{q} \leq c(n, p, r)\|D v\|_{L^{p}\left(\partial B_{s^{\prime}}, \mathbb{R}^{N \times n}\right)}^{p}\|v\|_{L^{r}\left(\partial B_{s^{\prime}}, \mathbb{R}^{N}\right)}^{q-p} .
$$

Therefore, by the definition of $E_{s, t}$, the previous estimate in particular gives

$$
\begin{aligned}
\left(\int_{\left.B_{t^{\prime} \backslash B_{s^{\prime}}}|\tilde{v}|^{q^{\star}} d x\right)^{\frac{q}{q^{\star}}}}+\int_{B_{t^{\prime}} \backslash B_{s^{\prime}}}|D \tilde{v}|^{q} \mathrm{~d} x\right. \\
\leq\|v\|_{L^{r}\left(\partial B_{s^{\prime}}, \mathbb{R}^{N}\right)}^{q-p}\left(\frac{C}{t-s} \int_{B_{t} \backslash B_{s}}|D v|^{p} \mathrm{~d} x\right) .
\end{aligned}
$$

On the other hand by Corollary 2.2 we also have that there exists a function $\tilde{w} \in W^{1, p}\left(\mathbb{R}^{n}, \mathbb{R}^{N}\right)$ such that

$$
\tilde{w}=w \quad \text { on } \quad \partial B_{t^{\prime}}
$$

and

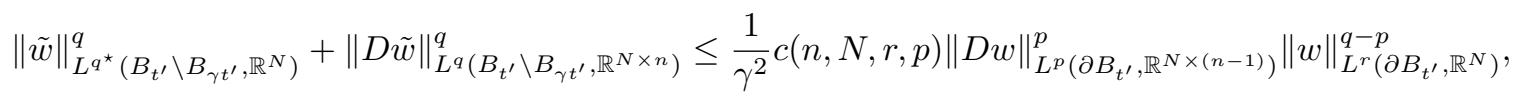


for every $\gamma \in(0,1)$. Hence, again by the definition of $E_{s, t}$, the previous estimate in particular yields

$$
\begin{aligned}
\left(\int_{\left.B_{t^{\prime} \backslash B_{s^{\prime}}}|\tilde{w}|^{q^{\star}} \mathrm{d} x\right)^{\frac{q}{q^{\star}}}}+\int_{B_{t^{\prime} \backslash B_{s^{\prime}}}|D \tilde{w}|^{q} \mathrm{~d} x} \leq\|w\|_{L^{r}\left(\partial B_{t^{\prime}}, \mathbb{R}^{N}\right)}^{q-p}\left(\frac{C}{t-s} \int_{B_{t} \backslash B_{s}}|D w|^{p} \mathrm{~d} x\right) .\right.
\end{aligned}
$$

Define the map

$$
z=\left\{\begin{array}{cl}
v & \text { on } B_{s^{\prime}} \\
\frac{\left(t^{\prime}-|x|\right) \tilde{v}+\left(|x|-s^{\prime}\right) \tilde{w}}{t^{\prime}-s^{\prime}} & \text { on } B_{t^{\prime}} \backslash B_{s^{\prime}} \\
w & \text { on } B_{1} \backslash B_{t^{\prime}} .
\end{array}\right.
$$

A direct computation shows that in $B_{t^{\prime}} \backslash B_{s^{\prime}}$

$$
|D z|^{q} \leq C\left(|D \tilde{v}|^{q}+|D \tilde{w}|^{q}+\frac{|\tilde{v}-\tilde{w}|^{q}}{\left(t^{\prime}-s^{\prime}\right)^{q}}\right) .
$$

Then combining inequality (2.30) with estimates (2.28), (2.29), using Hölder's inequality and the fact that $t^{\prime}-s^{\prime}>$ $\frac{t-s}{8}$, we easily get

$$
\begin{aligned}
\int_{B_{t^{\prime}} \backslash B_{s^{\prime}}}|D z|^{q} \mathrm{~d} x & \leq C \frac{t^{2}}{s^{2}(t-s)^{1+\frac{q(n-1)}{n}}}\|v\|_{L^{r}\left(\partial B_{s^{\prime}}, \mathbb{R}^{N}\right)}^{q-p} \int_{B_{t} \backslash B_{s}}|D v|^{p} \mathrm{~d} x \\
& +C \frac{t^{2}}{s^{2}(t-s)^{1+\frac{q(n-1)}{n}}}\|w\|_{L^{r}\left(\partial B_{t^{\prime}}, \mathbb{R}^{N}\right)}^{q-p} \int_{B_{t} \backslash B_{s}}|D w|^{p} \mathrm{~d} x .
\end{aligned}
$$

The other estimate follows similarly.

Remark 2.2. Notice that arguing in the same way we can connect two functions in $W^{1, p}\left(B_{1}(0), \mathbb{R}^{N}\right) \cap L^{\infty}\left(B_{1}(0), \mathbb{R}^{N}\right)$ or $W^{1, p}\left(B_{1}(0), \mathbb{R}^{N}\right) \cap B M O\left(B_{1}(0), \mathbb{R}^{N}\right)$ or in $W^{1, p}\left(B_{1}(0), \mathbb{R}^{N}\right) \cap C^{0, \alpha}\left(B_{1}(0), \mathbb{R}^{N}\right)$ with a function $W^{1, q}$ in a ring domain of $B_{1}(0)$, where $q$ is determined by Theorem 1.1.

In particular, when the two maps belong to $W^{1, n-1}\left(B_{1}(0), \mathbb{R}^{N}\right)$, the connection between them is a $W^{1, n}$ map in a ring domain of $B_{1}(0)$. In this case the regularity assumption on the traces is guaranteed by the borderline Sobolev embedding theorem. Namely, we have

Lemma 2.2. Let $v, w \in W^{1, n-1}\left(B_{1}(0), \mathbb{R}^{N}\right)$ and $0<s<t<1$. There exist a function $z \in W^{1, n}\left(B_{1}(0), \mathbb{R}^{N}\right)$ and $0<s<s^{\prime}<t^{\prime}<r<1$, depending on $v$, w such that

$$
\frac{t-s}{8} \leq t^{\prime}-s^{\prime} \leq t-s
$$

and

Moreover

$$
z= \begin{cases}v & \text { on } B_{s^{\prime}} \\ w & \text { on } B_{1} \backslash B_{t^{\prime}}\end{cases}
$$

$$
\begin{aligned}
\int_{B_{t^{\prime} \backslash B_{s^{\prime}}}|D z|^{n} \mathrm{~d} x \leq} & C \frac{t^{2}}{s^{2}(t-s)^{n+\frac{1}{n-1}}}\left(\int_{B_{t} \backslash B_{s}}|D v|^{n-1} \mathrm{~d} x\right)^{\frac{n}{n-1}} \\
& +C \frac{t^{2}}{s^{2}(t-s)^{n+\frac{1}{n-1}}}\left(\int_{B_{t} \backslash B_{s}}|D w|^{n-1} \mathrm{~d} x\right)^{\frac{n}{n-1}}
\end{aligned}
$$

and

$$
\begin{aligned}
\int_{B_{t^{\prime}} \backslash B_{s^{\prime}}}|z|^{n} \mathrm{~d} x \leq & C \frac{t^{2}}{s^{2}(t-s)^{\frac{n}{n-1}}}\left(\int_{B_{t} \backslash B_{s}}|D v|^{n-1} \mathrm{~d} x\right)^{\frac{n}{n-1}} \\
& +C \frac{t^{2}}{s^{2}(t-s)^{\frac{n}{n-1}}}\left(\int_{B_{t} \backslash B_{s}}|D w|^{n-1} \mathrm{~d} x\right)^{\frac{n}{n-1}}
\end{aligned}
$$

where $C=C(n, N)$. 


\section{A PARTIAL REgUlarity RESUlt}

In this section we shall prove the partial regularity result of Theorem 1.1. As usual, we shall use a linearization argument aimed to establish a decay estimate for the so called excess functional, that measures how the gradient of a local minimizer is far from being constant in a ball.

3.1. Caccioppoli type estimates. The aim of this section is to prove a Caccioppoli type inequality for minimizers of the rescaled functionals defined in Lemma 3.1 below. The inequality can be viewed as a perturbation of the classical Caccioppoli inequality. For our purposes the important point is that the additional terms do not affect the blow-up procedure.

We start by observing that the weak bound on the second derivative at (1.6), via the following result from [1], it suffices to control the growth of certain rescaled functionals that appear in our proof.

Lemma 3.1. Let $n \geq 2$ and let $G: \mathbb{R}^{N \times n} \rightarrow \mathbb{R}$ be an integrand of class $C^{2}$ satisfying

$$
|G(\xi)| \leq L\left(1+|\xi|^{n}\right) \quad \text { and } \quad|D G(\xi)| \leq c\left(1+|\xi|^{n-1}\right)
$$

Then for every $M>0$ there exists a constant $\beta=\beta(M)$ such that, if we define for $\lambda>0$ and $A \in \mathbb{R}^{N \times n}$ with $|A| \leq M$ the shifted and rescaled integrand

$$
G_{A, \lambda}=\frac{1}{\lambda^{2}}[G(A+\lambda \xi)-G(A)-\lambda D G(A) \xi]
$$

then

$$
\begin{aligned}
\left|G_{A, \lambda}(\xi)\right| & \leq \beta\left(|\xi|^{2}+\lambda^{n-2}|\xi|^{n}\right) \\
\left|D G_{A, \lambda}(\xi)\right| & \leq \beta\left(|\xi|+\lambda^{n-2}|\xi|^{n-1}\right) .
\end{aligned}
$$

Now, we are ready to prove the following

Theorem 3.1. Let $G_{A, \lambda}$ be a the function defined in (3.1) and let $v \in W_{\mathrm{loc}}^{1, n-1}\left(B_{1}(0), \mathbb{R}^{N}\right)$ be a local $G_{A, \lambda}-$ minimizer. Then there exists a positive constant $c=c(n, N, \beta, \nu)$ such that

$$
f_{B_{\tau}}\left(|D v|^{2}+\lambda^{n-3}|D v|^{n-1}\right) \mathrm{d} x \leq c \int_{B_{2 \tau}} \frac{|v|^{2}}{\tau^{2}} \mathrm{~d} x+c(\tau) \lambda^{\frac{2}{n-1}}\left(\lambda^{n-3} \int_{B_{2 \tau}}|D v|^{n-1} \mathrm{~d} x\right)^{\frac{n}{n-1}},
$$

for every $0<\tau<\frac{1}{2}$.

Proof. Fix $0<\frac{\tau}{2}<s<t<\tau<\frac{1}{2}$. By Lemma 2.2 there exist a function $z \in W^{1, n}\left(B_{1}(0), \mathbb{R}^{N}\right)$ and $s<s^{\prime}<t^{\prime}<t$, depending on $v$ such that

$$
\frac{t-s}{8} \leq t^{\prime}-s^{\prime} \leq t-s
$$

and

Moreover

$$
z= \begin{cases}v & \text { on } B_{s^{\prime}} \\ v & \text { on } B_{1} \backslash B_{t^{\prime}}\end{cases}
$$

$$
\int_{B_{t^{\prime}} \backslash B_{s^{\prime}}}|D z|^{n} \mathrm{~d} x \leq c \frac{1}{(t-s)^{n+\frac{1}{n-1}}}\left(\int_{B_{t} \backslash B_{s}}|D v|^{n-1} \mathrm{~d} x\right)^{\frac{n}{n-1}}
$$

and

$$
\int_{B_{t^{\prime}} \backslash B_{s^{\prime}}}|z|^{n} \mathrm{~d} x \leq c \frac{1}{(t-s)^{\frac{n}{n-1}}}\left(\int_{B_{t} \backslash B_{s}}|D v|^{n-1} \mathrm{~d} x\right)^{\frac{n}{n-1}},
$$

where $c=c(n, N)$. Fix now a cut-off function $\eta \in C_{0}^{\infty}\left(B_{t^{\prime}}\right)$, such that $\eta \equiv 1$ in $B_{s^{\prime}}$ and $|\nabla \eta| \leq \frac{c}{t^{\prime}-s^{\prime}}$. Since $\eta z \in W_{0}^{1, n-1}\left(B_{t^{\prime}}\right)$, the strict uniform quasiconvexity of $G$ stated in (H3), implies

$$
\nu \int_{B_{s^{\prime}}}\left(|D v|^{2}+\lambda^{n-3}|D v|^{n-1}\right) \mathrm{d} x \leq \int_{B_{t^{\prime}}} G_{A, \lambda}(D(\eta z)) \mathrm{d} x
$$




$$
\begin{aligned}
= & \int_{B_{s^{\prime}}} G_{A, \lambda}(D v) \mathrm{d} x+\int_{B_{t^{\prime}} \backslash B_{s^{\prime}}} G_{A, \lambda}(D(\eta z)) \mathrm{d} x \\
\leq & \int_{B_{t^{\prime}}} G_{A, \lambda}(D v) \mathrm{d} x-\int_{B_{t^{\prime}} \backslash B_{s^{\prime}}} G_{A, \lambda}(D v)+\beta \int_{B_{t^{\prime}} \backslash B_{s^{\prime}}}\left(|D(\eta z)|^{2}+\lambda^{n-2}|D(\eta z)|^{n}\right) \mathrm{d} x \\
\leq & \int_{B_{t^{\prime}}} G_{A, \lambda}(D((1-\eta) z)) \mathrm{d} x-\int_{B_{t^{\prime}} \backslash B_{s^{\prime}}} G_{A, \lambda}(D v) \mathrm{d} x \\
& +\beta \int_{B_{t^{\prime}} \backslash B_{s^{\prime}}}\left(|D(\eta z)|^{2}+\lambda^{n-2}|D(\eta z)|^{n}\right) \mathrm{d} x \\
\leq & \beta \int_{B_{t^{\prime}} \backslash B_{s^{\prime}}}\left(|D((1-\eta) z)|^{2}+\lambda^{n-2}|D((1-\eta) z)|^{n}\right) \mathrm{d} x \\
& +\beta \int_{B_{t^{\prime}} \backslash B_{s^{\prime}}}\left(|D(\eta z)|^{2}+\lambda^{n-2}|D(\eta z)|^{n}\right) \mathrm{d} x \\
& -\int_{B_{t^{\prime}} \backslash B_{s^{\prime}}} G_{A, \lambda}(D v) \mathrm{d} x \\
=: & I+I I+I I I .
\end{aligned}
$$

In previous estimate we used that $\eta z \equiv v$ on the ball $B_{s^{\prime}}$, the minimality of $v$ and the growth condition (3.2). Using the properties of $\eta$, we get

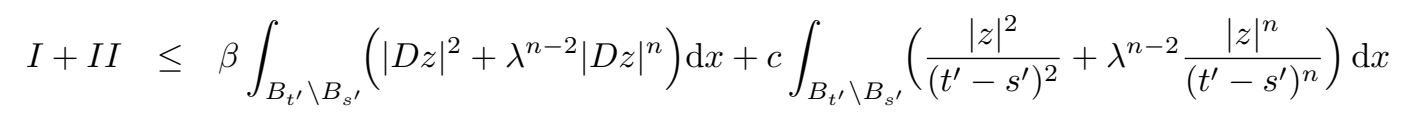

Reasoning as in Corollary 2.2, thanks to Proposition 2.1, we evaluate the $L^{2}$-norm of $D z$, as follows

$$
\int_{B_{t^{\prime}} \backslash B_{s^{\prime}}}|D z|^{2} \leq c \int_{B_{t} \backslash B_{s}}|D v|^{2}
$$

and

$$
\int_{B_{t^{\prime}} \backslash B_{s^{\prime}}}|z|^{2} \leq c \int_{B_{t} \backslash B_{s}}|v|^{2} .
$$

Using (3.3), (3.4), (3.6) and (3.7), we get

$$
\begin{aligned}
I+I I \leq & c \int_{B_{t} \backslash B_{s}}|D v|^{2} \mathrm{~d} x+c \int_{B_{\tau}} \frac{|v|^{2}}{(t-s)^{2}} \mathrm{~d} x \\
& +\frac{c}{(t-s)^{\frac{n^{2}}{n-1}}} \lambda^{n-2}\left(\int_{B_{\tau}}|D v|^{n-1} \mathrm{~d} x\right)^{\frac{n}{n-1}} .
\end{aligned}
$$

In order to estimate $I I I$, we recall that, by virtue of definition of $G$ at (3.1), we have

Therefore, we have

$$
G_{A, \lambda}(\xi)=\int_{0}^{1} \int_{0}^{1}\left\langle D^{2} G(A+s t \lambda \xi) s \xi, \xi\right\rangle d s d t
$$

$$
\begin{aligned}
I I I= & -\int_{\left(B_{t^{\prime}} \backslash B_{s^{\prime}}\right) \cap\{|\lambda D v| \leq 1\}} G_{A, \lambda}(D v)-\int_{\left(B_{t^{\prime}} \backslash B_{s^{\prime}}\right) \cap\{|\lambda D v|>1\}} G_{A, \lambda}(D v) \\
= & -\int_{\left(B_{t^{\prime}} \backslash B_{s^{\prime}}\right) \cap\{|\lambda D v| \leq 1\}} \int_{0}^{1} \int_{0}^{1}\left\langle D^{2} G(A+s t \lambda D v) s D v, D v\right\rangle d s d t \mathrm{~d} x \\
& -\frac{1}{\lambda^{2}} \int_{\left(B_{t^{\prime}} \backslash B_{s^{\prime}}\right) \cap\{|\lambda D v|>1\}} G(A+\lambda D v)-G(A)-D G(A) \lambda D v \\
\leq & k_{M} \int_{\left(B_{t} \backslash B_{s}\right) \cap\{|\lambda D v| \leq 1\}}|D v|^{2} \\
& +\frac{1}{\lambda^{2}} \int_{\left(B_{t} \backslash B_{s}\right) \cap\{|\lambda D v|>1\}}|G(A)|+|D G(A) \| \lambda D v|
\end{aligned}
$$




$$
\begin{aligned}
& \leq k_{M} \int_{\left(B_{t} \backslash B_{s}\right)}|D v|^{2} \mathrm{~d} x+\frac{1}{\lambda^{2}} \int_{\left(B_{t} \backslash B_{s}\right) \cap\{|\lambda D v|>1\}}(|G(A)|+|D G(A)|) \lambda^{2}|D v|^{2} d x \\
& \leq k_{M} \int_{B_{t} \backslash B_{s}}|D v|^{2} .
\end{aligned}
$$

Inserting (3.8) and (3.9) in (3.5), we get

$$
\begin{aligned}
& \nu \int_{B_{s}}\left(|D v|^{2}+\lambda^{n-3}|D v|^{n-1}\right) \mathrm{d} x \leq \nu \int_{B_{s^{\prime}}}\left(|D v|^{2}+\lambda^{n-3}|D v|^{n-1}\right) \mathrm{d} x \\
\leq & c(n, M) \int_{B_{t} \backslash B_{s}}|D v|^{2} \mathrm{~d} x+c \int_{B_{\tau}} \frac{|v|^{2}}{(t-s)^{2}} \mathrm{~d} x \\
& +\frac{c}{(t-s)^{\frac{n^{2}}{n-1}}} \lambda^{n-2}\left(\int_{B_{\tau}}|D v|^{n-1} \mathrm{~d} x\right)^{\frac{n}{n-1}}
\end{aligned}
$$

We fill the hole by adding to both sides of (3.10) the term

thus obtaining

$$
c(n, M) \int_{B_{s}}\left(|D v|^{2}+\lambda^{n-3}|D v|^{n-1}\right) \mathrm{d} x
$$

$$
\begin{aligned}
& \int_{B_{s}}\left(|D v|^{2}+\lambda^{n-3}|D v|^{n-1}\right) \mathrm{d} x \\
\leq & \theta \int_{B_{t}}\left(|D v|^{2}+\lambda^{n-3}|D v|^{n-1}\right) \mathrm{d} x+c \int_{B_{\tau}} \frac{|v|^{2}}{(t-s)^{2}} \mathrm{~d} x \\
& +\frac{c}{(t-s)^{\frac{n^{2}}{n-1}}} \lambda^{n-2}\left(\int_{B_{\tau}}|D v|^{n-1} \mathrm{~d} x\right)^{\frac{n}{n-1}}
\end{aligned}
$$

with $\theta<1$. A standard iteration Lemma (see [29]) yields

$$
\int_{B_{\frac{\tau}{2}}}\left(|D v|^{2}+\lambda^{n-3}|D v|^{n-1}\right) \mathrm{d} x \leq c \int_{B_{\tau}} \frac{|v|^{2}}{\tau^{2}} \mathrm{~d} x+c(\tau) \lambda^{\frac{2}{n-1}}\left(\lambda^{n-3} \int_{B_{\tau}}|D v|^{n-1} \mathrm{~d} x\right)^{\frac{n}{n-1}} .
$$

We conclude the proof, dividing previous inequality by $\left|B_{\tau}\right|$.

3.2. The decay estimate. As usual, to get the partial regularity result stated in Theorems 1.1, it suffices to have a decay estimate for the excess function $U\left(x_{0}, r\right)$ defined as

$$
U\left(x_{0}, r\right)=f_{B_{r\left(x_{0}\right)}}\left(\left|D u-(D u)_{x_{0}, r}\right|^{2}+\left|D u-(D u)_{x_{0}, r}\right|^{n-1}\right) \mathrm{d} x .
$$

The desired decay estimate is established in the next Proposition.

Proposition 3.1. Assume that $u \in W_{\mathrm{loc}}^{1, p}(\Omega)$ is a local minimizer for an integrand $G: \mathbb{R}^{N \times n} \rightarrow \mathbb{R}$ satisfying hypotheses (H1), (H2), (H3).

Fix $M>0$. There exists a constant $C_{M}>0$ such that for every $0<\tau<\frac{1}{4}$ there exists $\varepsilon=\varepsilon(\tau, M)$ with the following property. If

then

$$
\left|(D u)_{x_{0}, r}\right| \leq M \quad \text { and } \quad U\left(x_{0}, r\right) \leq \varepsilon
$$

Proof. Fix M and $\tau$. We shall determine $C_{M}$ later. We argue by contradiction assuming that there exists a sequence of balls $B_{r_{h}}\left(x_{h}\right)$ satisfying

$$
B_{r_{h}}\left(x_{h}\right) \subset \Omega, \quad\left|(D u)_{x_{h}, r_{h}}\right| \leq M, \quad \lim _{h} U\left(x_{h}, r_{h}\right)=0,
$$

but

$$
U\left(x_{h}, \tau r_{h}\right)>C_{M}^{*} \tau^{2} U\left(x_{h}, r_{h}\right)
$$


Set

$$
A_{h}=(D u)_{x_{h}, r_{h}} \quad a_{h}=(u)_{x_{h}, r_{h}} \quad \lambda_{h}^{2}=U\left(x_{h}, r_{h}\right)
$$

Step 1 (Blow up): We rescale the function $u$ in each $B_{r_{h}}\left(x_{h}\right)$ to obtain a sequence of functions on $B_{1}=$ $B_{1}(0)$. Set

$$
v_{h}(y)=\frac{1}{\lambda_{h} r_{h}}\left[u\left(x_{h}+r_{h} y\right)-a_{h}-r_{h} A_{h} y\right], \quad y \in B_{1} .
$$

Then $v_{h} \in W^{1, p}\left(B_{1}\right)$ and

$$
D v_{h}(y)=\frac{1}{\lambda_{h}}\left[D u\left(x_{h}+r_{h} y\right)-A_{h}\right] .
$$

Clearly we have

$$
\left(D v_{h}\right)_{0,1}=0, \quad\left|A_{h}\right| \leq M,
$$

and

$$
\frac{U\left(x_{h}, r_{h}\right)}{\lambda_{h}^{2}}=f_{B_{1}}\left(\left|D v_{h}\right|^{2}+\lambda_{h}^{n-3}\left|D v_{h}\right|^{n-1}\right) \mathrm{d} y=1 .
$$

Then, passing possibly to a subsequence, we may suppose that

$$
v_{h} \rightarrow v \quad \text { weakly in } W^{1,2}\left(B_{1}\right)
$$

and

$$
A_{h} \rightarrow A \text {. }
$$

Step 2 (v solves a linear system): Now we show that

$$
\int_{B_{1}(0)} \frac{\partial^{2} G}{\partial \xi_{\alpha}^{i} \partial \xi_{\beta}^{j}}(A) D_{\beta} v^{j} D_{\alpha} \phi^{i} d y=0 \quad \forall \phi \in C_{0}^{1}\left(B_{1}, \mathbb{R}^{N}\right) .
$$

The minimizer $u$ is a weak solution to the Euler-Lagrange system (see Remark 1.1). Then, rescaling in each $B_{r_{h}}\left(x_{h}\right)$, we get for any $\phi \in C_{0}^{1}\left(B_{1}, \mathbb{R}^{N}\right)$ and each $1 \leq i \leq N$

$$
\int_{B_{1}} \frac{\partial G}{\partial \xi_{\alpha}^{i}}\left(A_{h}+\lambda_{h} D v_{h}\right) D_{\alpha} \phi^{i} d y=0,
$$

and therefore

$$
\frac{1}{\lambda_{h}} \int_{B_{1}(0)}\left[\frac{\partial G}{\partial \xi_{\alpha}^{i}}\left(A_{h}+\lambda_{h} D v_{h}\right)-\frac{\partial G}{\partial \xi_{\alpha}^{i}}\left(A_{h}\right)\right] D_{\alpha} \phi^{i} \mathrm{~d} y=0 .
$$

Let us write

$$
B_{1}=E_{h}^{+} \cup E_{h}^{-}=\left\{y \in B_{1}: \lambda_{h}\left|D v_{h}(y)\right|>1\right\} \cup\left\{y \in B_{1}: \lambda_{h}\left|D v_{h}(y)\right| \leq 1\right\},
$$

then by (3.14) we get

$$
\left|E_{h}^{+}\right| \leq \int_{E_{h}^{+}} \lambda_{h}^{2}\left|D v_{h}\right|^{2} d y \leq \lambda_{h}^{2} \int_{B_{1}(0)}\left|D v_{h}\right|^{2} d y \leq c \lambda_{h}^{2} .
$$

Now, by (H4), we have that

$$
\begin{aligned}
& \frac{1}{\lambda_{h}}\left|\int_{E_{h}^{+}}\left[D G\left(A_{h}+\lambda_{h} D v_{h}\right)-D G\left(A_{h}\right)\right] D \phi d y\right| \\
\leq & \left(\frac{c}{\lambda_{h}}\left|E_{h}^{+}\right|+c \lambda_{h}^{n-2} \int_{E_{h}^{+}}\left|D v_{h}\right|^{n-1} \mathrm{~d} y\right)\|D \phi\|_{L^{\infty}} \\
\leq & c \lambda_{h}\|D \phi\|_{L^{\infty}},
\end{aligned}
$$

where we used (3.14) again. From this it follows that

$$
\lim _{h} \frac{1}{\lambda_{h}} \int_{E_{h}^{+}}\left[D G\left(A_{h}+\lambda_{h} D v_{h}\right)-D G\left(A_{h}\right)\right] D \phi \mathrm{d} y=0 .
$$


On $E_{h}^{-}$we have

$$
\begin{aligned}
& \frac{1}{\lambda_{h}} \int_{E_{h}^{-}}\left[D G\left(A_{h}+\lambda_{h} D v_{h}\right)-D G\left(A_{h}\right)\right] D \phi \mathrm{d} y \\
= & \int_{E_{h}^{-}} \int_{0}^{1} D^{2} G\left(A_{h}+s \lambda_{h} D v_{h}\right) D v_{h} D \phi \mathrm{d} s \mathrm{~d} y \\
= & \int_{E_{h}^{-}} \int_{0}^{1}\left[D^{2} G\left(A_{h}+s \lambda_{h} D v_{h}\right)-D^{2} G\left(A_{h}\right)\right] D v_{h} D \phi \mathrm{d} s \mathrm{~d} y \\
& +\int_{E_{h}^{-}} D^{2} G\left(A_{h}\right) D v_{h} D \phi \mathrm{d} y .
\end{aligned}
$$

Note that (3.19) ensures that $\chi_{E_{h}^{-}} \rightarrow \chi_{B_{1}}$ in $L^{r}\left(B_{1}\right)$ for all $r<\infty$ and by (3.14) we have,

$$
\lambda_{h} D v_{h}(y) \rightarrow 0 \quad \text { in measure on } \quad B_{1} .
$$

Then, by (3.15), (3.16) and the uniform continuity of $D^{2} F$ on bounded sets, we get

$$
\begin{gathered}
\lim _{h} \frac{1}{\lambda_{h}} \int_{E_{h}^{-}}\left[D G\left(A_{h}+\lambda_{h} D v_{h}\right)-D G\left(A_{h}\right)\right] D \phi \mathrm{d} y \\
=\int_{B_{1}} D^{2} G(A) D v D \phi \mathrm{d} y .
\end{gathered}
$$

By (3.18), (3.21) and the above equality, we infer that $v$ solves system (3.17). Since $D^{2} F(A)$ satisfies the Legendre-Hadamard condition by $(\mathrm{H} 3)$, we can apply the classical regularity results for the solution $v$ (see for example [29]). Consequently, for any $0<\tau<1 / 2$, we have

$$
f_{B_{\tau}}\left|D v-(D v)_{\tau}\right|^{2} \mathrm{~d} y \leq c \tau^{2} f_{B_{1}}\left|D v-(D v)_{1}\right|^{2} \mathrm{~d} y \leq c \tau^{2}
$$

and

$$
\left|(D v)_{2 \tau}-(D v)_{\tau}\right|^{2} \leq c \tau^{2}
$$

where $c=c(M)$. Moreover we have

$$
v \in C^{\infty}\left(B_{1}, \mathbb{R}^{N}\right)
$$

Step 3 (Conclusion): We set

$$
G_{A_{h}, \lambda_{h}}(\xi)=\frac{1}{\lambda_{h}^{2}}\left[G\left(A_{h}+\lambda_{h} \xi\right)-G\left(A_{h}\right)-\lambda_{h} D G\left(A_{h}\right) \xi\right]
$$

and for every $r<1$

$$
I_{h, r}(w)=\int_{B_{r}} G_{A_{h}, \lambda_{h}}(D w) d y .
$$

One can easily check that $v_{h}$ minimizes the functional $I_{h, r}$ for every $h$.

Fix $\tau \in\left(0, \frac{1}{4}\right)$, set $b_{h}=\left(v_{h}\right)_{B_{2 \tau}}, B_{h}=\left(D v_{h}\right)_{B_{\tau}}$ and define

$$
w_{h}(y)=v_{h}(y)-b_{h}-B_{h} y .
$$

Note that $w_{h}$ minimizes

$$
J_{h, r}(w)=\int_{B_{r}} \tilde{G}_{h}(D w) \mathrm{d} y
$$

where

$$
\tilde{G}_{h}(\xi)=\frac{1}{\lambda_{h}^{2}}\left[G\left(A_{h}+\lambda_{h}\left(\xi+B_{h}\right)\right)-G\left(A_{h}+\lambda_{h} B_{h}\right)-\lambda_{h} G\left(A_{h}+\lambda_{h} B_{h}\right) \xi\right]
$$


We have that $\tilde{G}_{h}$ satisfies the inequalities in (3.2) of Lemma 3.1, for some constant that could depend on $\tau$ through $\left|\lambda_{h} B_{h}\right|$. But, given $\tau \in\left(0, \frac{1}{4}\right)$, we may always choose $h$ large enough to have $\left|\lambda_{h} B_{h}\right|<\frac{\lambda_{h}}{\tau^{\frac{n}{2}}}<1$. Then we can apply to $w_{h}$ the estimate of Theorem 3.1 , thus obtaining

$$
\begin{aligned}
\frac{U\left(x_{h}, \tau r_{h}\right)}{\lambda_{h}^{2}} & =f_{B_{\tau}}\left(\left|D w_{h}\right|^{2}+\lambda_{h}^{n-3}\left|D w_{h}\right|^{n-1}\right) \mathrm{d} y \\
& \leq c f_{B_{2 \tau}} \frac{\left|w_{h}\right|^{2}}{\tau^{2}} \mathrm{~d} y+c(\tau) \lambda_{h}^{\frac{2}{n-1}}\left(\lambda_{h}^{n-3} \int_{B_{2 \tau}}\left|D w_{h}\right|^{n-1} \mathrm{~d} y\right)^{\frac{n}{n-1}}
\end{aligned}
$$

Now, passing to the limit as $h \rightarrow \infty$ in (3.23), using Poincaré's inequality and estimate (3.22) we get

$$
\begin{aligned}
\lim _{h} \frac{U\left(x_{h}, \tau r_{h}\right)}{\lambda_{h}^{2}} & \leq \frac{c}{\tau^{2}} f_{B_{2 \tau}}\left(\left|v-(v)_{2 \tau}-(D v)_{2 \tau} y\right|^{2} \mathrm{~d} y+\frac{c}{\tau^{2}} f_{B_{2 \tau}}\left|(D v)_{2 \tau}-(D v)_{\tau}\right|^{2} y^{2} \mathrm{~d} y\right. \\
& \left.\leq c f_{B_{2 \tau}}\left|(D v)_{2 \tau}-(D v)_{\tau}\right|^{2}\right) \mathrm{d} y \\
& \leq C_{M} \tau^{2}
\end{aligned}
$$

which contradicts (3.13) if we choose $C_{M}^{*}$ larger than $C_{M}$.

It follows from our proof that the singular set can be explicitly described as follows:

$$
\begin{gathered}
\Omega \backslash \Omega_{0} \subseteq\left\{x \in \Omega: \liminf _{r \rightarrow 0} f_{B(x, r)}\left|V(D u)-V\left((D u)_{x, r}\right)\right|^{2}>0\right. \text { or } \\
\left.\limsup _{r \rightarrow 0}\left|V\left((D u)_{x, r}\right)\right|=\infty\right\} .
\end{gathered}
$$

\section{A nONLINEAR $\mathcal{A}$-HARMONiC EXTENSION}

Let $\Omega \subset \mathbb{R}^{n}$ be a $C^{2}$ bounded domain. Another application of our main result is an $\mathcal{A}$-harmonic extension of Sobolev functions defined on $\partial \Omega$.

More precisely, let us consider $\mathcal{A}: \Omega \times \mathbb{R}^{N \times n} \mapsto \mathbb{R}^{N \times n}$ a continuous mapping satisfying, for $m, \ell, L$ positive constants, a parameter $\mu \geq 0$ and an exponent $p \geq 2$, the following conditions

$$
\begin{aligned}
|\mathcal{A}(x, z)-\mathcal{A}(y, z)| & \leq m\left(\mu^{2}+|z|^{2}\right)^{\frac{p-1}{2}}|x-y| \\
z \mapsto \mathcal{A}(x, z) \text { is } C^{1} \text { and }(x, z) & \mapsto D_{z} \mathcal{A}(x, z) \text { is jointly continuous } \\
\left|D_{z} \mathcal{A}(x, z)\right| & \leq L\left(\mu^{2}+|z|^{2}\right)^{\frac{p-2}{2}} \\
\left\langle D_{z} \mathcal{A}(x, z) \lambda, \lambda\right\rangle & \geq \ell\left(\mu^{2}+|z|^{2}\right)^{\frac{p-2}{2}}|\lambda|^{2}
\end{aligned}
$$

for every $z, \lambda \in M$ and a.e. $x \in \Omega$.

Let us consider the boundary value problem

$$
\left\{\begin{aligned}
-\operatorname{div} \mathcal{A}(x, D u) & =0 & & \text { in } \Omega \\
u & =f & & \text { on } \partial \Omega
\end{aligned}\right.
$$

where $f \in W^{1, p}\left(\partial \Omega ; \mathbb{R}^{N}\right)$. It is well known by the Browder-Minty theory of monotone operators that problem (P) admits a unique solution $u \in W^{1, p}\left(\Omega ; \mathbb{R}^{N}\right)$. Let

$$
\mathcal{S}_{\mathcal{A}}: W^{1, p}\left(\partial \Omega ; \mathbb{R}^{N}\right) \mapsto W^{1, p}\left(\Omega ; \mathbb{R}^{N}\right)
$$

denote the solution operator. The aim of this section is to prove the following 
Theorem 4.1. Let $\mathcal{A}$ satisfy the assumption (C1)-(C4). Then we have the following three statements

k) If $f \in W^{1, p}\left(\partial \Omega ; \mathbb{R}^{N}\right) \cap L^{r}\left(\partial \Omega, \mathbb{R}^{N}\right)$ with $\frac{(n-1) p}{n-1-p} \leq r<+\infty$ then

$$
\left\|D \mathcal{S}_{\mathcal{A}}(f)\right\|_{L^{q}\left(\Omega, \mathbb{R}^{N \times n}\right)}^{q} \leq c(\Omega, n, N, p, r, m, \ell, L)\|D f\|_{L^{p}\left(\partial \Omega, \mathbb{R}^{N \times(n-1)}\right)}^{p}\|f\|_{L^{r}\left(\partial \Omega, \mathbb{R}^{N}\right)}^{q-p},
$$

where $q=\min \left\{p+\frac{r}{n-1+r}, \frac{p n}{n-2}\right\}$.

kk) If $f \in W^{1, p}\left(\partial \Omega ; \mathbb{R}^{N}\right) \cap \in B M O\left(\partial \Omega, \mathbb{R}^{N}\right)$ then

$$
\left\|D \mathcal{S}_{\mathcal{A}}(f)\right\|_{L^{q}\left(\Omega, \mathbb{R}^{N \times n}\right)}^{q} \leq c(\Omega, n, N, p, m, \ell, L)\|D f\|_{L^{p}\left(\partial \Omega, \mathbb{R}^{N \times(n-1)}\right)}^{p}\|f\|_{B M O\left(\partial \Omega, \mathbb{R}^{N}\right)},
$$

where $q=\min \left\{p+1, \frac{p n}{n-2}\right\}$.

kkk) If $f \in W^{1, p}\left(\partial \Omega ; \mathbb{R}^{N}\right) \cap C^{0, \alpha}\left(\partial \Omega, \mathbb{R}^{N}\right)$ then,

$$
\left\|D \mathcal{S}_{\mathcal{A}}(f)\right\|_{L^{q}\left(\Omega, \mathbb{R}^{N \times n}\right)}^{q} \leq c(\Omega, n, N, p, \alpha, m, \ell, L)\|D f\|_{L^{p}\left(\partial \Omega, \mathbb{R}^{N \times(n-1)}\right)}^{p}\|f\|_{C^{0, \alpha}\left(\partial \Omega, \mathbb{R}^{N}\right)}^{\frac{1}{1-\alpha}},
$$

where $q=\min \left\{p+\frac{1}{1-\alpha}, \frac{p n}{n-2}\right\}$.

Proof. Suppose that $\Omega=B_{R}$. We will only give details for the statement $k$ ), the others can be proven analogously. Since $f \in W^{1, p}\left(\partial \Omega ; \mathbb{R}^{N}\right) \cap L^{r}\left(\partial \Omega, \mathbb{R}^{N}\right)$, by using Corollary 2.2, we get a function $\tilde{f} \in W^{1, q}\left(B_{R} \backslash B_{\frac{R}{4}}\right)$, with $q=p+\frac{r}{n-1+r}$, such that

$$
\|D \tilde{f}\|_{L^{q}\left(B_{R} \backslash B_{\frac{R}{4}}, \mathbb{R}^{N \times n}\right)}^{q} \leq c(n, N, p, r)\|D f\|_{L^{p}\left(\partial B_{R}, \mathbb{R}^{N \times n}\right)}^{p}\|f\|_{L^{r}\left(\partial B_{R}, \mathbb{R}^{N}\right)}^{q-p} .
$$

Now let us define

$$
F=\left\{\begin{array}{cl}
0 & \text { on } B_{\frac{R}{4}} \\
\frac{|x|-R / 4}{R / \tilde{f}} \tilde{f} & \text { on } B_{\frac{R}{2}} \backslash B_{\frac{R}{4}} \\
\tilde{f} & \text { on } B_{R} \backslash B_{\frac{R}{2}} .
\end{array}\right.
$$

By (4.4), one can easily check that $F \in W^{1, q}\left(B_{R} ; \mathbb{R}^{N}\right)$ and that

$$
\|D F\|_{L^{q}\left(B_{R}, \mathbb{R}^{N \times n}\right)}^{q} \leq c(n, N, p, r, R)\|D f\|_{L^{p}\left(\partial B_{R}, \mathbb{R}^{N \times n}\right)}^{p}\|f\|_{L^{r}\left(\partial B_{R}, \mathbb{R}^{N}\right)}^{q-p} .
$$

Since $\mathcal{S}_{\mathcal{A}}(f)=\mathcal{S}_{\mathcal{A}}(F)$, by applying Theorem 7.8 in [33] we have that $\mathcal{S}_{\mathcal{A}}(f) \in W^{1, q}\left(B_{R}\right)$, for every $q \leq$ $\min \left\{p+\frac{r}{n-1+r}, \frac{p n}{n-2}\right\}$ and the following estimate

$$
\left\|D \mathcal{S}_{\mathcal{A}}(f)\right\|_{L^{q}\left(B_{R}, \mathbb{R}^{N \times n}\right)} \leq C(R, n, N, q, m, \ell, L)\|D F\|_{L^{q}\left(B_{R}, \mathbb{R}^{N \times n}\right)}
$$

holds. Combining previous estimate with (4.5) gives the conclusion on balls. The result for general $C^{2}$ domains, follows through a standard covering argument.

\section{REFERENCES}

[1] E. Acerbi and N. Fusco. A regularity theorem for minimizers of quasiconvex integrals. Arch. Ration. Mech. Anal. 99 (1987), 261-281.

[2] E. Acerbi and N. Fusco. Partial regularity under an isotropic $(p, q)$ growth conditions. J. Diff. Eq. 107 (1994), $46-67$.

[3] J.M. Ball and F. Murat. $W^{1, p}$ quasiconvexity and variational problems for multiple integrals. J. Funct. Anal. 58 (1984), $225-253$.

[4] M. Bildhauer. Convex variational problems. Linear, nearly linear and anisotropic growth conditions. Lecture Notes in Mathematics, 1818. Springer-Verlag, Berlin, 2003.

[5] M. Bildhauer and M. Fuchs. Higher integrability of the gradient for vectorial minimizers of decomposable variational integrals. Manuscripta Math. 123 (2007), 269-283.

[6] M. Bildhauer, M. Fuchs and X. Zhou. A regularity theory for scalar local minimizers of splitting-type variational integrals. Ann. Sc. Norm. Super. Pisa Cl. Sci. (5) 6 (2007), no. 3, 385-404.

[7] M. Carozza, N. Fusco and G. Mingione. Partial regularity of minimizers of quasiconvex integrals with subquadratic growth. Ann. Mat. Pura e Appl. 175, 4 (1998), 141-164.

[8] M. Carozza, J. Kristensen and A. Passarelli di Napoli. Higher differentiability of minimizers of convex variational integrals. Ann. I. H. Poincaré AN 28 (2011), 395-411. 
[9] M. Carozza, J. Kristensen and A. Passarelli di Napoli. Regularity of minimizers of autonomous convex variational integrals. Ann. Sc. Norm. Super. Pisa Cl. Sci. (5) 13 (2014), no. 4, 1065-1089.

[10] M. Carozza, J. Kristensen and A. Passarelli di Napoli. On the validity of the Euler-Lagrange system. Commun. Pure Appl. Anal. 14 (2015), no. 1, 51-62.

[11] M. Carozza and A. Passarelli di Napoli. A regularity theorem for quasiconvex integrals: the case $1<p<2$. Proc. of The Royal Soc. of Edinburgh 126A (1996), 1181-1199.

[12] M. Carozza and A. Passarelli di Napoli. Partial regularity for anisotropic functionals of higher order. ESAIM COCV 13,4 (2007), 692706.

[13] M. Carozza and A. Passarelli di Napoli. Model problems from nonlinear elasticity: partial regularity results. ESAIM COCV 13,1 (2007), $120-134$.

[14] C.Y. Chen and J. Kristensen. On coercive variational integrals. Nonlinear Anal. 153 (2017), 213-229.

[15] E. De Giorgi: Un esempio di estremali discontinue per un problema variazionale di tipo ellittico. Boll. Un. Mat. Ital., 4, 1 135-137, 1968.

[16] De Maria, B., Passarelli di Napoli, A.: Partial regularity for non autonomous functionals with non standard growth conditions. Calc. Var. Partial Differential Equations 38 (2010), no. 3-4, 417-439.

[17] L. Esposito and G. Mingione. Partial regularity for minimizers of degenerate polyconvex energies. J. Convex Anal. 8,1 (2001), 1-38.

[18] L. Esposito, F. Leonetti and G. Mingione. Regularity results for minimizers of irregular integrals with $(p, q)$ growth. Forum Mathematicum 14 (2002), 245-272

[19] L. Esposito, F. Leonetti and G. Mingione. Sharp regularity for functionals with ( $p, q)$ growth. J. Diff. Eq. 204 (2004), 5-55.

[20] L.C. Evans. Quasiconvexity and partial regularity in the calculus of variations. Arch. Ration. Mech. Anal. 95 (1986), $227-252$.

[21] I. Fonseca and N. Fusco. Regularity results for anisotropic image segmentation models. Ann. Sc. Norm. Super. Pisa 24 (1997), $463-499$.

[22] I. Fonseca and J. Malý. Relaxation of multiple integrals in Sobolev spaces below the growth exponent for the energy density. Ann. I.H.Poincaré (Anal. Nonlineare) 14 (1997), 309-338.

[23] I. Fonseca and J. Malý. From Jacobian to Hessian: distributional form and relaxation. Riv. Mat. Univ. Parma 7 (2005), Proc. Conf. "New trends in Calculus of Variations", E. Acerbi and G. Mingione (Eds.), 45-74.

[24] N. Fusco and J.E. Hutchinson. Partial regularity and everywhere continuity for a model problem from non-linear elasticity. J. Austral. Math. Soc. (Series A) 57 (1994), 158-169.

[25] N. Fusco and J.E. Hutchinson. Partial regularity in problems motivated by non-linear elasticity. SIAM J. Math. Anal. 22 (1991), 15161551.

[26] M. Giaquinta. Multiple integrals in the calculus of variations and nonlinear elliptic systems. Annals of Math. Studies 105, Princeton Univ. Press, 1983.

[27] M. Giaquinta. Growth conditions and regularity, a counterexample. Manuscripta Math. 59 (1987), $245-248$.

[28] M. Giaquinta, G. Modica. Partial regularity of minimizers of quasiconvex integrals. Ann. Inst. H. Poincaré 3 (1986), 185-208

[29] E. Giusti. Metodi diretti in calcolo delle variazioni. U.M.I. (1994)

[30] F. Gmeineder and J. Kristensen. Partial regularity for BV minimizers. Arch. Ration. Mech. Anal. 232 (2019), no. 3, $1429-1473$.

[31] J. Kristensen. Lower semicontinuity of quasi-convex integrals in BV. Calc. Var. Partial Diff. Eq. 7 (1998), $249-261$.

[32] J. Kristensen. A necessary and sufficient condition for lower semicontinuity. Nonlinear Anal. 120 (2015), 43-56.

[33] J. Kristensen and G. Mingione. The Singular Set of Minima of Integral Functionals. Arch. Ration. Mech. Anal. 180 (2006), $331-398$.

[34] F. Leonetti. Maximum principles for vector-valued minimizers of some integral functionals. Boll. Un. Mat. Ital. A (6) 5 (1991), 51-56.

[35] J. Malý. Weak lower semicontinuity of polyconvex integrals. Proc. Roy. Soc. Edinb. 123A (1993), 681-691.

[36] P. Marcellini. Approximation of quasiconvex functions and lower semicontinuity of multiple integrals. Manuscripta Math. 51 (1986), 1-28.

[37] P. Marcellini. Un example de solution discontinue d' un probéme variationel dans le cas scalaire. Preprint Ist. U.Dini, Firenze, 1987-88.

[38] P. Marcellini. Regularity of minimizers of integrals of the calculus of variations with non-standard growth conditions. Arch. Ration. Mech. Anal. 105 (1989), 267-284.

[39] P. Marcellini. Regularity and existence of solutions of elliptic equations with ( $p, q)$ growth conditions. J. Diff. Eq. 90 (1991), 1-30.

[40] P. Marcellini. Everywhere regularity for a class of elliptic systems without growth conditions. Ann. Scuola Normale Sup. Pisa, Cl. Sci. 23 (1996), 1-25.

[41] C. Mooney and O. Savin. Some singular minimizers in low dimensions in the calculus of variations. Arch. Ration. Mech. Anal. 221 (2016), no. 1, 1-22.

[42] C.B. Morrey, Jr. Multiple integrals in the Calculus of Variations. Springer-Verlag, 1966.

[43] J. Nečas. Example of an irregular solution to a nonlinear elliptic system with analytic coefficients and conditions for regularity. Theor. Nonlin. Oper., Constr. Aspects, Proc. int. Summer Sch., Berlin 1975, 197-206 (1977).

[44] A. Passarelli di Napoli and F. Siepe. A regularity result for a class of anisotropic systems. Rend. Ist. Mat di Trieste (1997), 13-31.

[45] T. Schmidt. Regularity of minimizers of $W^{1, p}$-quasiconvex variational integrals with $(p, q)$-growth. Calc. Var. Partial Differential Equations. 32,1 (2008), 1-24

[46] T. Schmidt. Regularity of relaxed minimizers of quasiconvex variational integrals with $(p, q)$-growth. Arch. Ration. Mech. Anal. 193,2 (2009), 311-337.

[47] T. Schmidt. Regularity theorems for degenerate quasiconvex energies with $(p, q)$-growth. Adv. Calc. Var. 1,3 (2008), 241-270.

[48] V. Šverák and X. Yan. Non-Lipschitz minimizers of smooth uniformly convex functionals. Proc. Natl. Acad. Sci. USA 99, 15269-15276 (2002). 


\section{Menita Carozza}

Dipartimento di Ingegneria - Università degli Studi del Sannio

Corso Garibaldi- 82100 Benevento, Italy

E-mail address: carozza@unisannio.it

\section{Jan Kristensen}

Mathematical Institute, University of Oxford, Andrew Wiles Building,

Oxford OX2 6GG, United Kingdom

E-mail address: kristens@maths.ox.ac.uk

Antonia Passarelli di Napoli

Dipartimento di Mat. e Appl. 'R. Caccioppoli', Università degli Studi di Napoli "Federico II"

Via Cintia, 80126 Napoli, Italy

E-mail address: antpassa@unina.it 